\section{USING BIOMARKERS TO MEASURE WORK STRESS: ALLOSTATIC LOAD BY OCCUPATION AND INDUSTRY IN THE CHILEAN WORKFORCE}

Devan Hawkins, Laura Kernan, Junhee Cho, Manuel Cifuentes. University of Massachusetts Lowell, Lowell, MA, USA

10.1136/oemed-2014-102362.276

Objectives One hypothesised mechanism by which chronic stress results in negative health outcomes is through allostatic load (AL), which is a measure of the cumulative 'wear and tear' experienced by the body when activating physiologic responses in order to maintain homeostasis. This studied aimed to quantify and compare the mean levels of allostatic load experienced by workers in different occupations and industries in Chile.

Method From a weighted national survey of the Chilean population $(\mathrm{n}=1199)$, occupations and industries were categorised by their average AL level, which was measured using a composite of secondary biomarkers of chronic stress exposure that fell outside of a "well-centred" range (extreme normal high value biomarkers). The numbers of biomarkers that fell outside of this range were counted for each worker in order to represent AL. Adjusting by age, sex, education, smoking status, and personal income, Poisson-log generalised linear mixed models were used to generate mean levels of AL for each occupation and industry. Results An important and statistically significant gradient was observed in mean AL levels between different occupations (from 0.7 to 4.1 mean number of extreme normal high value biomarkers) and industries (from 0.8 to 2.3 ).

Conclusions There is a clear occupational gradient of AL in the Chilean workforce. Preventive and clinical activities should focus on workers of those occupations with highest AL, because previous studies have observed an association between $\mathrm{AL}$ and mortality. The work-related risk factors that generate these occupational and industrial gradients in AL should be examined further.

\section{BENZENE OCCUPATIONAL EXPOSURE ESTIMATES IN BRAZIL USING A JOB-EXPOSURE MATRIX}

Maria Juliana Moura-Correa, Vilma Santana. Federal University of Bahia, Salvador, Bahia, Brazil

\subsection{6/oemed-2014-102362.277}

Objectives To estimate the number and prevalence of occupational exposure to benzene in Brazil.

Method This study was carried out with the economic active population of Brazil, using 2010 Census data. Benzene data from the Finisth National Job-Exposure Matrix, FINJEM, by occupational groups and sex were used to estimate the weighted number and prevalence of occupational exposure to benzene in Brazil. Because of differences in the Brazilian and Finish occupational classification codes, an assessment of acceptance by occupational safety and health experts was also made.

Results From a total of 86353839 workers, 7376761 have jobs in occupations potentially exposed to benzene. Based on FINJEM parameters, approximately 778025 workers were identified as probable exposed to benzene in their jobs, a weighted prevalence of 9.1/1000 workers, higher among men (11.2/1000) than among women (6.0/1000). Most of the benzene exposed workers were from the group of machine repair and engine mechanics (66\%). The nine experts who ranked the occupational codes regrouping agreed with $97 \%$ of the proposed matches.
Conclusions Benzene is targeted by health surveillance in Brazil but little data are available on occupational exposure. Job-exposure matrix can be an useful tool for epidemiological monitoring of benzene exposure for surveillance purpose. There is a need to develop a JEM with national data thus making feasible the evaluation of the Benzene National Agreement impact on this exposure control and workers' health.

\section{DEPRESSION AND ANXIETY AS AN OUTCOME OF JOB STRAIN IN THE CHILEAN WORKFORCE}

Laura Kernan, Junhee Cho, Devan Hawkins, Manuel Cifuentes. University of Massachusetts Lowell, Lowell MA, USA

\subsection{6/oemed-2014-102362.278}

Objectives Based on previous analyses, using ROC curves and correlation, we aim to improve agreement assessments between diverse formulations of the Demand Control (DC) Model for Job Strain in order to test its ability to predict anxiety and depression $(\mathrm{AD})$ in a nationally representative population of workers from Chile, a country transitioning to high economic development and with high prevalence of AD.

Method A weighted national sample of 9503 workers representing the entire Chilean workforce was surveyed during 20102011 in Chile. Goldberg Health Questionnaire (12 questions) to assess $\mathrm{AD}$ and diverse formulation of the DC model were used as dependent and independent variables respectively. Bland-Altman plots for agreement and Poisson-log models (controlled for demographics) for predictive ability were used to assess each formulation.

Results Good agreement between Log and Quotient formulations. For different formulations, high strain jobs had between 1.7 (quadrant and tertile formulations) and 3.7 (extreme tertile formulation), higher prevalence of AD than low strain jobs. Approximately 12 $25 \%$ of $\mathrm{AD}$ cases might be attributed to increased strain.

Conclusions Predictive ability of the DC model for AD was similar in trend to other studies. Most accurate models (extreme formulations) represent less population and might be impractical. At the cost of excluding many people from the evaluation, the extreme tertile model seems to be the best formulation to predict $\mathrm{AD}$ among extreme exposures to job strain within the Chilean working population. Better operationalizations of the DC model should be considered in future surveys to allow international comparisons and guide eventual interventions.

\section{LONG-TERM STYRENE EXPOSURE IMPAIRS COLOUR VISION IN FIBREGLASS REINFORCED PLASTICS LAMINATORS}

${ }^{1}$ Chi-Hsien Chen, ${ }^{2}$ Po-Chen Hong, ${ }^{3}$ Ya-Fen Wang, ${ }^{4}$ Perng-Jy Tsai, ${ }^{1}$ Yue Leon Guo. ${ }^{1}$ Department of Environmental and Occupational Medicine, National Taiwan University Hospital, Taipei, Taiwan; 'Division of Occupational Medicine, Institute of Occupational Safety and HealthCouncil of Labor Affairs, Executive Yuan, Taipei, Taiwan; ${ }^{3}$ Department of Bioenvironmental Engineering, Chung Yuan Christian University, Chung-Li, Taiwan; ${ }^{4}$ Department of Occupational Safety and Health, College of Public Health, China Medical University, Taichung, Taiwan

\subsection{6/oemed-2014-102362.279}

Objectives Although styrene has been reported to cause colour vision impairment, the results were still inconclusive. Whether the impairment was related to short-term or long-term exposure was not known. The study aims to evaluate colour vision in high styrene exposed fibreglass-reinforced plastics (FRP) laminators. 
Method Fifty workers from two yacht factories participated, including 23 FRP laminators and 27 non-FRP workers. Pre-shift colour confusion index (CCI) was measured on the first workday of a week by using Lanthony Desaturated Panel D-15d. Concentrations of volatile organic compounds related to FRP laminating were collected by a stainless canister, and then analysed using a GC/MS. Cumulative working hours for FRP laminating was obtained from daily administrative records of the companies. Logistic regression was used to assess correlation between cumulative working hours (past $0.5,2$, and 8 years) of FRP laminating and pre-shift CCI, where work time and CCI were stratified by median and the model was adjusted for age and regular alcohol consumption.

Results The mean styrene exposure during FRP laminating was $4.2 \mathrm{ppm}$. The median of cumulative working hours for FRP laminating in the past $0.5,2$ and 8 years was $2,49,248 \mathrm{~h}$ respectively. Higher cumulative working hours for FRP laminating was associated with poor CCI (past 0.5 yrs, odd ratio $(\mathrm{OR})=3.1, \mathrm{p}$ $=0.1$; past 2 yrs, $\mathrm{OR}=4.8, \mathrm{p}=0.03$; past 8 yrs, $\mathrm{OR}=6.5, \mathrm{p}=$ $0.01)$. The effect of long-term exposure to styrene appeared to be stronger than short-term exposure.

Conclusions Long-term exposure to styrene from FRP laminating was associated with colour vision impairment.

\section{HEAD AND NECK CANCER AND OCCUPATIONAL EXPOSURE TO ASBESTOS, MINERAL WOOLS AND SILICA: RESULTS FROM THE ICARE STUDY}

${ }^{1}$ Sophie Paget-Bailly, 'Diane Cyr, 'Matthieu Carton, ${ }^{1}$ Florence Guida, ${ }^{1}$ Isabelle Stucker, ${ }^{2}$ Danièle Luce. ${ }^{1}$ INSERM U1018, Villejuif, France, ${ }^{2}$ INSERM U1085, Pointe À Pitre, France

\subsection{6/oemed-2014-102362.280}

Objectives To study the associations between head and neck cancer risk and occupational exposure to asbestos, mineral wools and silica.

Method ICARE is a population based case-control study conducted in France. Analyses were restricted to men and included 1833 cases of head and neck squamous cell carcinomas (HNSCC) and 2747 controls. Complete occupational history was collected. Occupational exposures were assessed through job-exposure matrices. Logistic models were used to estimate adjusted odds ratios (ORs) and 95\% confidence intervals (CI).

Results Exposure to asbestos was associated with an elevated risk of HNSCC $(\mathrm{OR}=1.9$, CI 1.6-2.3), and the risk increased significantly with the probability, duration and cumulative level of exposure. Significantly increased risks were found for all cancer sites: larynx $(\mathrm{OR}=2.1, \mathrm{CI} 1.6-2.8)$, hypopharynx $(\mathrm{OR}=2.0$ CI 1.5-2.8), oropharynx $(\mathrm{OR}=1.6 \mathrm{CI}=1.3-2.1)$ and oral cavity $(\mathrm{OR}=1.9$ CI 1.4-2.6). Conversely, after adjustment for asbestos exposure, exposure to mineral wools was not associated with HNSCC risk $(\mathrm{OR}=0.8 \mathrm{CI} 0.6-1.0)$, for any of the cancer sites. Exposure to silica was not associated with HNSCC risk overall $(\mathrm{OR}=0.9$ CI $0.7-1.2)$, but non-significantly elevated ORs were observed for the highest level of cumulative exposure for oropharyngeal $(\mathrm{OR}=1.6 \mathrm{CI} 0.8-3.2)$ and hypopharyngeal cancer $(\mathrm{OR}=1.9$ CI 0.9-4.1).

Conclusions Our findings confirm the role of asbestos exposure in laryngeal cancer, and suggest that asbestos exposure increases also the risk of oral and pharyngeal cancer. There is some evidence of an association between silica exposure and pharyngeal cancer. Exposure to mineral wools was not associated with HNSCC risk in our study.

\section{ESTIMATING THE PROPORTION OF OCCUPATIONAL CANCERS WITH MINIMAL RESOURCES: AN EXAMPLE FROM QUEBEC}

1,2 France Labrèche, ${ }^{1}$ Patrice Duguay, ${ }^{1}$ Alexandre Boucher, ${ }^{3}$ Robert Arcand. ${ }^{1}$ Institut de Recherche Robert-Sauvé en Santé Et en Sécurité Du Travail (IRSST), Montreal, Quebec, Canada; '2School of Public Health, Université de Montréal, Montreal, Quebec, Canada; ${ }^{3}$ Institut National de Santé Publique Du Québec, Montreal, Quebec, Canada

\subsection{6/oemed-2014-102362.281}

Objectives To estimate the number of work-related cancer cases and deaths in order to prioritise research activities.

Method Numbers of compensated incident cancers (between 2005-2007) and cancer deaths (between 1997-2005) were obtained from the Quebec Workers' Compensation Board. A second series of estimates was calculated by applying proportions of cancers attributable to work published for Finland (Nurminen and Karjalainen 2001) and for the United Kingdom (Rushton and colleagues 2012) to Quebec tumour registry data for 28 cancer sites. A comparison of industrial profiles of Finland, United Kingdom and Canada showed reasonable similarities between the countries over the last decades.

Results Compensation statistics reported an annual average of 94 incident cancers and 40 cancer deaths (98-99\% men), 60$64 \%$ of which being mesotheliomas, followed by respiratory cancers (30-37\%). Using published estimates of attributable fractions, it was estimated that $6.0 \%$ of incident cancers (men, 9.1\%; women, $2.7 \%$ ) and $7.6 \%$ of cancer deaths (men, 11.7\%; women, $2.8 \%$ ) could be attributable to work, resulting annually in 2200 new cancers and 1200 deaths. Incident cancers of the lungs, prostate, bladder, skin and breast (women) were the most numerous, whereas cancer sites resulting in more deaths were lung, breast (women) and mesothelium. On average, 53\% of incident mesothelioma cases were compensated yearly.

Conclusions This attempt at better estimating, albeit imperfectly, importance of the burden of cancer from occupational exposures can help prioritise research activities and increase stakeholders' awareness. However, better estimates of human impact and economic costs are warranted to justify large investments in preventive interventions.

\section{PSYCHOSOCIAL IMPACTS OF A CONTAMINATED NEEDLESTICK INJURY OR BLOOD AND BODY FLUID EXPOSURE IN HEALTH CARE WORKERS}

1,2Hsueh-Ching Wu, ${ }^{3}$ Weishan Chin, ${ }^{2}$ Judith Shu-Chu Shiao, ${ }^{3,4}$ Yue-Liang Leon Guo, ${ }^{5}$ Yueh-Tzu Hsieh. ${ }^{1}$ Department of Nursing, Hsin Sheng Junior College of Medical Care and Management, Taoyuan, Taiwan; 'Department of Nursing, College of Medicine, National Taiwan University (NTU) and NTU Hospital, Taipei, Taiwan; ${ }^{3}$ Institute of Occupational Medicine and Industrial Hygiene, College of Public Health, National Taiwan University, Taipei, Taiwan; ${ }^{4}$ Department of Environmental and Occupational Medicine, College of Medicine, National Taiwan University (NTU) and NTU Hospital, Taipei, Taiwan; ${ }^{5}$ Department of Nursing, Chung Hwa University of Medical Technology, Tainan, Taiwan

\subsection{6/oemed-2014-102362.282}

Objectives This study aims to explore the psychosocial impacts of health care workers (HCW) who were exposed to a contaminated needlestick Injury (NSI) or blood and body fluid (BBF) at work.

Method Personal interviews were performed using a phenomenological approach including in-depth interviews fort data collection, and expert meetings for data analysis. The 